\title{
Incorporation of Hydroxyapatite and Calcium Triphosphate in the Epoxy Resin-Based Sealer
}

\author{
Ahmed Adel Al-Quraini ${ }^{10}$ Abdulrahman Fahad Al-Aodah ${ }^{20}$ Adam Ahmed M. Al-Qadhi ${ }^{30}$ \\ Abdul Majeed Munir Ahmad ${ }^{4}(0$
}

${ }^{1}$ College of Dentistry, King Faisal University, Al Hofuf, Saudi Arabia

2 Royal Commission Health Services, Al-Jubail, Saudi Arabia

3 Dental Hospital King Faisal University, Al Hofuf, Saudi Arabia

${ }^{4}$ College of Dentistry, Imam Abdulrahman Bin Faisal University,

Dammam, Saudi Arabia

Eur Dent Res Biomater J 2021;2:47-51.

\author{
Address for correspondence Ahmed Adel Al-Quraini, College of \\ Dentistry, King Faisal University, Al Hofuf, Saudi Arabia \\ (e-mail: aalquraini@kfu.edu.sa).
}

\begin{abstract}
Keywords

- bond strength

- endodontics

- hydroxyapatite

- nanoparticles

- root canal treatment

Objective This study aims to assess and compare the influence of hydroxyapatite (HA) and tricalcium phosphate (TCP) nanoparticles on a commercially available epoxy resinbased sealer, focusing on porosity and push-out bond strength.

Materials and Methods This work was classified into a control group and two experimental groups. In each experimental group, the sealer was mixed with $2.5 \mathrm{wt}$. $\%$ of HA and TCP nanoparticles. Thirty extracted single-rooted teeth were utilized. After sectioning the crowns, the remaining roots of 15 teeth were used, up to 40 to 0.06 , using a $\mathrm{K} 3$ rotary system. Smear layers were removed with $3 \mathrm{~mL}$ of $17 \%$ EDTA applied for 60 seconds. Then, the canals were irrigated with $3 \mathrm{~mL}$ of $2.25 \% \mathrm{NaOCl}$ and $5 \mathrm{~mL}$ of distilled water. The strength of push-out bonds was tested via an Instron universal testing machine on a $2 \mathrm{~mm}$ section acquired from obturated canals. Data were assessed using one-way ANOVA and post hoc tests at a significance level of $p<0.05$. Results A nonsignificant difference $(p>0.05)$ was evident when the three groups were crosschecked in terms of void volume and bond strength. Micro-CT evaluations revealed the lowest volume of voids to be $0.1152 \mathrm{~mm}^{3}$ (2.69\%) for the HA group compared with the control group $0.1818 \mathrm{~mm}^{3}(3.9 \%)$ and the TCP group $0.2194 \mathrm{~mm}^{3}$ (4.33\%). Mean bond strength values were $4.18 \pm 1.77 \mathrm{MPa}$ for group 1 (control), $4.19 \pm 1.54 \mathrm{MPa}$ for group 2 (HA 2.5\%) and $3.76 \pm 1.95 \mathrm{MPa}$ for group 3 (TCP 2.5\%). Groups 1 and 3 showed both cohesive and a mixed type of failure, while group 2 showed adhesive and a mixed type of bond failure.

Conclusion Within the limitations of the study, incorporation of $2.5 \mathrm{wt} \% \mathrm{HA}$ and TCP nanoparticles into AH Plus did not significantly affect the percentage volume of voids and the bond strength negatively.
\end{abstract}

published online

December 13, 2021
DOI https://doi.org/

$10.1055 / \mathrm{s}-0041-1739398$. ISSN 2791-7452. (c) 2021. The Author(s).

This is an open access article published by Thieme under the terms of the Creative Commons Attribution License, permitting unrestricted use, distribution, and reproduction so long as the original work is properly cited. (https://creativecommons.org/licenses/by/4.0/)

Thieme Medical and Scientific Publishers Pvt. Ltd., A-12, 2nd Floor, Sector 2, Noida-201301 UP, India 


\section{Introduction}

In endodontics, the root canal is the process of removing the infected pulp and restoring it with an appropriate filling material. ${ }^{1,2}$ Gutta-percha has been used as a core root filling material for more than 100 years. $^{3}$ However, gutta-percha alone fails to provide three-dimensional sealing, as it cannot adhere to the canal walls, leaving gaps and allowing access for bacteria. ${ }^{4}$ Therefore, a pertinent objective following a root canal therapy is attaining a flawless seal to diminish bacterial spread, subsequently denigrating any chances of pulpal pathology. ${ }^{5}$ A sealer acts as a synergist in establishing a hermetic seal along with an obturating material. Second, a sealer needs to be biocompatible, as it is close to periapical tissue and has supercilious dimensional stability. ${ }^{6}$ Various categories of root canal sealers have been introduced in the market and are being used in clinical practice. Zinc oxide-, epoxy resin-, methacrylate-, calcium hydroxide-, silicon-, bioceramic-, glass ionomer-, and MTA-based sealers are the major categories of commercially available sealers. Due to their inherent weak structural characteristics, they do not meet the requirements of an ideal root canal sealer ${ }^{6}$; however, epoxy resin sealers gained global attention due to their exceptional dislodging resistance, as a strong covalent bond is formed between $\mathrm{AH}$ plus and epoxide rings of amino acids exhibited at the dentin-collagen interface. Epoxy resins showed promising results in the complex and inherently wet environment of chemo-mechanically cleaned and shaped root canal systems, where they do not shrink following installation. ${ }^{7}$ Moreover, epoxy resin sealers also bind to the collagen in the wall of the root canal. ${ }^{6}$

It is clinically beneficial to utilize the proven advantages of an epoxy resin sealer with some desired properties of hydroxyapatite (HA) and tricalcium phosphate (TCP) materials. The structure of HA is identical to that of dentine and enamel; hence, it can adhere strongly to the dentinal tubules. Furthermore, literature signifies its biocompatibility and reactivity characteristics. ${ }^{8,9}$ While calcium and phosphate ions are potential candidates to augment tooth remineralization and roots' strength, dentin hardness is achieved when $\mathrm{Ca}$ and $\mathrm{P}$ ions, in the sealer, neutralizes the acidic environment. ${ }^{10}$ Commercial sealers, constituting calcium phosphate or HA have been developed due to their ability to influence healing and bone regeneration.

As dictated by professional obligation, researchers and dental practitioners are constantly endeavoring on bringing innovations in biomaterials to improve the beneficial effects. This present study aims to evaluate the effect of the incorporation of HA and tricalcium phosphate nanoparticles into a commercially available epoxy resin-based sealer on porosity and push-out bond strength. Push-out tests are attaining widespread popularity in experimental studies due to their capacity of characterizing the behavior of restorations or sealers at the interface. ${ }^{11}$

\section{Materials and Methods}

Hydroxyapatite (HA) and TCP powders were obtained from the Interdisciplinary Research Center in Biomedical Materials, COMSATS IIT, Pakistan. AH Plus (Dentsply, Germany) was
Table 1 Details of the samples used in the study for comparative analysis

\begin{tabular}{|l|l|}
\hline Groups & Sealers \\
\hline G1 (Control) & AH Plus \\
\hline G2 (Experimental) & AH Plus +2.5 wt.\% HA \\
\hline G3 (Experimental) & AH Plus +2.5 wt.\% TCP \\
\hline
\end{tabular}

used as a control endodontic sealer. This study comprised a control group and two experimental groups according to the wt.\% of particles at $2.5 \%$. The details of the three groups are presented in - Table 1.

\section{Tooth Preparation}

For this study, 30 extracted single-rooted human teeth were acquired. They were cleaned of remaining tissue and calculus, disinfected in $1 \%$ chloramine for 24 hours. Then, they were stored in a $1 \%$ thymol solution at $4^{\circ} \mathrm{C}$. Afterward, the teeth with curved roots, caries, cracks, open apices, or previous root canal treatments were omitted. Later, all teeth were decoronated using a high-speed carbide bur and water spray. Fifteen teeth were used for obturation, and the remaining teeth were used to obtain root dentin sections for bond strength.

Fifteen roots of approximately equal size $10 \pm 1 \mathrm{~mm}$ were selected. Each canal of the 15 roots was accessed, and the working length was determined by subtracting $1 \mathrm{~mm}$ from the length of an inserted \#15 K-file (Dentsply Maillefer, Ballaigues, Switzerland) with its tip visualized at the apical foramen. The canals were operated on using a K3 Rotary System, 0.06 taper rotary instruments (Kerr Dental, USA) to a master apical file size \#40. Between each file size, the canal was irrigated with $3 \mathrm{~mL}$ of $2.5 \%$ sodium hypochlorite $(\mathrm{NaOCL})$ using a 27-G side-vented needle. After completion of the root canal preparation, the smear was removed by rinsing each root canal with $3 \mathrm{~mL}$ of $17 \%$ EDTA (UltraDent, Germany) for 60 seconds, followed by rinsing with $3 \mathrm{~mL}$ of $2.25 \% \mathrm{NaOCl}$. A final flush was performed with $5 \mathrm{~mL}$ of distilled water. Then, the canal was dried with multiple sterile paper points. Teeth were then sporadically classified into three groups $(n=5)$.

\section{Obturation of Canals}

The prepared canals were covered with a thin layer of sealer and obturated with a 40;0.02 taper master cone and 20;0.02 taper accessory cones using the later compaction technique. All root fillings were performed by an experienced endodontist to standardize the procedure. Following the late condensation, GP was cut using a heated plastic instrument flush with the coronal surface and condensed vertically with a hand plugger. A layer of respective sealer preparation was then placed to obtain a coronal seal.

Control Group: Roots were filled using an AH Plus sealer dispensed using an auto-mixing tip.

HA Group: In this group, roots were filled with an AH Plus sealer containing $2.5 \mathrm{wt}$.\% HA nanoparticles. 
TCP Group: In this group, roots were filled with an AH Plus sealer containing $2.5 \mathrm{wt}$.\% TCP nanoparticles.

Weight measurements for the sealers, HA, and TCP were performed up to 4 decimal points using a pre-calibrated electronic weighing balance. $^{12}$

\section{Porosity}

Porosity was determined by measuring the variations in the percentage volume of voids between the two experimental groups using a high-resolution Micro-CT (SkyScan 1176, Bruker-microCT, Kontich, Belgium). After canal preparation and root filling, the teeth were scanned. The scanning procedures were conducted at an isotropic voxel size of $9 \mu \mathrm{m}$ using $50 \mathrm{kV}, 800 \mu \mathrm{A}, 360^{\circ}$ of the rotation angle, and a $0.5-\mathrm{mm}$ thick aluminum filter. The reconstructed images were assayed using the CTAn software (Brukermicro CT, Kontich, Belgium).

\section{Push-out Bond Strength}

To assess the bond strength, the remaining 15 roots were embedded in a self-cure acrylic resin and were sectioned transversally into 2-mm thick discs via a diamond saw (Isomet; Buehler, Lake Bluff, NY, USA). Two sections were acquired from the middle third of each root.

The canal of each section was widened to a standardized size with the pass of \#5 Gates Glidden Drill providing a canal space of $1.3 \mathrm{~mm}$. The sections were washed with $1 \mathrm{~mL}$ of $\mathrm{NaOCl}$ followed by a 1-minute application of $17 \%$ EDTA. Finally, the sections were washed with $5 \mathrm{~mL}$ of distilled water. Then, they were blot dried. The sealers were mixed as described above and introduced into the canal spaces avoiding air entrapment. Eight sections were used for each group. Sections were preserved in $80 \%$ relative humidity at $37^{\circ} \mathrm{C}$ for 24 hours to allow the initial setting of the sealer. Then, specimens were placed in artificial saliva $(\mathrm{pH} 7.0)$ at $37^{\circ} \mathrm{C}$. After a week, all sections were removed and a push-out test was performed at a crosshead speed of $1 \mathrm{~mm} / \mathrm{min}$ via a universal testing machine (Instron, Instron Ltd., High Wycombe, England). The maximum load at failure $\left(\mathrm{F}_{\max }\right)$ was tallied in $\mathrm{N}$. The bonding surface A was gauged through the following equation:

$$
\mathrm{A}=2 \pi r \times h,
$$

Where $\pi$ represents the constant $3.14, r$ the root canal space radius, and $h$ the slice thickness in $\mathrm{mm}$. Afterward, the bond strength, denoted in MPa, was represented via the following $\mathrm{Eq}^{13}$ :

$$
\text { Bond Strength }(\mathrm{MPa})=\frac{\text { Debonding force }(\mathrm{N})}{\text { Bonded surface area }\left(\mathrm{mm}^{2}\right)} \text {. }
$$

\section{Statistical Analysis}

Data were analyzed with an NCSS 2007 (NCSS 2007, NCSS, LLC, Kaysville, UT, USA) using one-way analysis of variance
(ANOVA) and post hoc multiple comparison tests at a significance level of $p<0.05$.

\section{Results}

Micro-CT evaluations revealed the lowest volume of voids $0.1152 \mathrm{~mm}^{3}$ (2.69\%) for the HA group compared with the control group $0.1818 \mathrm{~mm}^{3}$ (3.9\%) and TCP group $0.2194 \mathrm{~mm}^{3}$ (4.33\%). However, the variances were not statistically significant $(p>0.05)$. All groups showed internal, external, and combined voids.

Mean bond strength values were $4.18 \pm 1.77 \mathrm{MPa}$ for the control group, $4.19 \pm 1.54 \mathrm{MPa}$ for the HA group (HA 2.5\%), and $3.76 \pm 1.95 \mathrm{MPa}$ for the TCP group $2.5 \%$. Incorporation of the $2.5 \%$ TCP nanoparticles in the AH Plus resulted in lesser values of bond strength compared with the other groups. Yet, there were no significant differences across the control and experimental groups $(p>0.05)$. The control and the TCP groups showed cohesiveness and a mixed type of failure, while the HA group showed only a mixed type of bond failure (-Table 2). - Fig. 1 shows the box-and-whisker plots for the bond strength of all three groups.

\section{Discussion}

This study's prospect was devised to assess the influence of HA and TCP nanoparticles when incorporated into an epoxy

Table 2 Nature of bond failure for all three groups

\begin{tabular}{|l|l|l|l|}
\hline Groups & $\begin{array}{l}\text { Nature of } \\
\text { bond } \\
\text { failure }\end{array}$ & Cohesive & Mixed \\
\cline { 2 - 3 } & Adhesive & & \\
\hline G1; AH Plus (Control) & - & 57 & 43 \\
\hline G2; AH Plus + 2.5\% HA & 15 & - & 85 \\
\hline G3; AH Plus + 2.5\% TCP & - & 28.5 & 71.5 \\
\hline
\end{tabular}

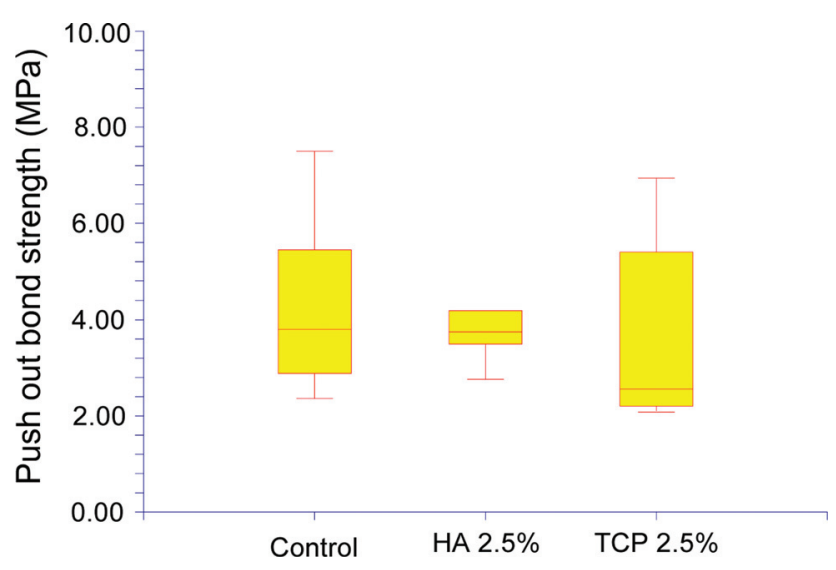

Fig. 1 Box-and-whisker plot showing the push-out bond strength values for control and experimental groups. The top and bottom lines indicate the maximum and minimum values in megapascals (MPa).

The box represents $75 \%$ of the values, and the line in the box indicates the median value. 
resin-based sealer. The outcomes were evaluated fortified on their sealing ability, bioactivity, and push-out bond strength. The core materials and the sealer should develop a stable, chemically bonded mass bonded to the dentine to keep leakage to a minimum. For root canal filling, it is optimal that the entire root canal space is completely filled, devoid of any gaps. Employing sealers as standalone components is not clinically preferable for canal filling. However, in our study, it was used solely, without incorporating any other filling material to determine the separate measurement of the $\mathrm{D} / \mathrm{S}$ interface bond strength. Also, three sealers were handpicked: the AH Plus, the critically acclaimed epoxy resinbased sealer, also known as the gold standard. ${ }^{14}$ Other sealers containing calcium phosphate and HA were used as well.

Tricalcium phosphate-based sealers have been garnering attention due to the bioactivity of the TCP phase associated with them. ${ }^{6}$ There is a potential that the interaction with dentine fluid could induce bio-mineralization with the formation of mineral plugs within the dentinal tubules, inspiring biological activity within the root canal. Tricalcium phosphate-based sealers may enhance the root fillings' seal through the in situ expression of their biological activity. The mineral tags were prominent, even in the current study, as a band of strength was shown in regions where the AH Plus penetrated the dentinal tubules. The literature specifies that incorporation of $\mathrm{ca}$ and $\mathrm{p}$ ions in resins aids in the regeneration of the lost minerals, thus augmenting root dentin's mechanical properties. Moreover, it was documented that hydroxyapatite addition influenced an increase in crystal growth, enhancing the process of mineralization. ${ }^{15}$ Regarding the push-out bond strength amongst the three resin sealers, TCP 2.5\% AH Plus exhibited the lowest values of pushout bond strength. Contralateral teeth provided a more balanced specimen selection, eliminating sample bias. The classical sealing ability testing methods showed nonsignificant variances among the experimental and control groups. This study found a difference in the mode of failure between AH Plus + TCP 2.5 wt., AH Plus + HA 2.5 wt.\%, in which the TCP and AH plus showed cohesiveness and a mixed type of failure, while the HA group showed an adhesive and a mixed type of bond failure. The results of our study agreed with the findings of Haddad et al, who found that the highest bond strength was in the gutta-percha coated with calcium phosphate, while the failure mode observed in his study was in a mixed-mode. ${ }^{13}$ In another study, Bae et al demonstrated that sealers constituting calcium phosphate showed enhanced bone regeneration. Osteoblast differentiation markers were observed in these sealers; they are essential for the process of mineralization. ${ }^{16}$ Baras et al documented an increase in dentin hardness after utilizing nano-sized particles of amorphous calcium phosphate in sealers. ${ }^{17}$

HA is a natural product, ${ }^{18}$ forming 60 to $70 \%$ of the calcified skeleton and the main bone components; thus, its integration into sealers is biologically sustainable and allows new bone formation, hence replacing the extruded material. Osteointegration, along with osteoconduction, may occur when HA is kept in contact with the bone. Moreover, it is known to have periapical healing properties that take place through forming a biological seal in the apical region, availing an intact closure. ${ }^{8}$ Witjaksono et al. designed an in vitro analysis to evaluate the sealing ability of HA in combination with epoxy resins as sealers. It was concluded that finer and lower average leakage values of HA in contrast to $\mathrm{ZnO}$ and $\mathrm{CaOH}$ base sealers took place due to HA's superior binding ability with viable bones. ${ }^{19}$ Toledano et al documented that HA nanoparticles, when used in combination with zinc oxide, strengthens the radicular dentin, since it enhances the process of remineralization, subsequently resulting in nano hardness. ${ }^{20}$ One could argue that HA might have acted as a filler and, in response, the restoration resisted the dislodgement forces as a unit.

Mainly, due to air entrapment, structural defects may result when a sealer is mixed or transferred. Moreover, physical characteristics which include density and rate of flow also account for these deficiencies. During setting, huge polymerization stresses result in the formation of a gap as the resin is released from the wall of the canal. ${ }^{21}$ Voids propagate crack formation and microleakage of fluids along with acids, demineralizing the structure of HA crystals. Consequently, fracture or recurrent caries may result. ${ }^{22,23}$ Cavity configuration factor (C-Factor) may evaluate the flow quantity of resin during the polymerization process. ${ }^{21}$ High values of C-Factor are found in long canals, as an inadequate area is provided for the relief of polymerization shrinkage stresses, thus debonding at the interface may result. The results revealed a nonsignificant difference among the three groups when compared for internal, external, and combined voids through micro-CT scanning. In a study by Akman et al in 2010, AH plus demonstrated 50\% void-free interfaces in contrast to Epiphany and MetaSEAL, which showed $50 \%$ and $70 \%$ void-free interface, respectively, from apical to coronal portions. Thus, this demonstrates their high adhesion ability. ${ }^{21}$ In another study, Mohammadian documented better adaptation of AH Plus sealer in contrast to ZOE or silicon sealers. ${ }^{24}$ So, it can be concluded that incorporation of $2.5 \mathrm{wt} . \%$ of HA or TCP in our study did not diminish the sealing ability of the sealer, and any voids formed might be caused by air or cavity defects.

To assess the interfaces' mechanical properties, the thin slice push-out test is an important experimental tool. ${ }^{11}$ The benefits of this method are evident in tensile and shear strength tests because it is less sensitive to small variations amongst specimens and in stress distribution during application of load, Besides, it is achievable to align samples for testing. ${ }^{25}$

\section{Conclusion}

Within the limitations of the study, incorporation of $2.5 \mathrm{wt} . \%$ HA and TCP nanoparticles into AH Plus did not significantly affect the percentage volume of voids and the bond strength negatively. Further long-term studies are required to observe the effects of incorporation of HA and TCP on the bond strength, bioactivity, and apatite formation. Utilizing a greater concentration of HA and TCP nanoparticles might be useful to get a significantly greater bond strength. 


\section{Conflict of Interest}

None declared.

\section{References}

1 Shahi S, Rahimi S, Yavari HR, et al. Effects of various mixing techniques on push-out bond strengths of white mineral trioxide aggregate. J Endod 2012;38(04):501-504

2 Siddiqui FA, Sheikh A, Rashid S. Radiographic evaluation of dentin thickness and pulp space width for different age groups. J Pak Dent Assoc 2012;1(02):99-102

3 Moorer WR, Genet JM. Antibacterial activity of gutta-percha cones attributed to the zinc oxide component. Oral Surg Oral Med Oral Pathol 1982;53(05):508-517

4 Razavian H, Barekatain B, Shadmehr E, Khatami M, Bagheri F, Heidari F. Bacterial leakage in root canals filled with resin-based and mineral trioxide aggregate-based sealers. Dent Res J (Isfahan) 2014;11(05):599-603

5 Alenzi A, Samran A, Samran A, et al. Restoration strategies of endodontically treated teeth among dental practitioners in Saudi Arabia. A nationwide pilot survey. Dent J (Basel) 2018;6(03):44

6 Tyagi S, Mishra P, Tyagi P. Evolution of root canal sealers: an insight story. Eur J Gen Dent 2013;2(03):199-218

7 Lottanti S, Tauböck TT, Zehnder M. Shrinkage of backfill guttapercha upon cooling. J Endod 2014;40(05):721-724

8 Masudi S, Luddin N, Mohamad D, Alkashakhshir J, Adnan R, Ramli R, Eds. In vitro Study on Apical Sealing Ability of NanoHydroxyapatite-Filled Epoxy Resin Based Endodontic Sealer. Presented in: AIP Conference Proceedings; 2010: American Institute of Physics

9 Khurshid Z, Zafar MS, Hussain S, Fareed A, Yousaf S, Sefat F. Silversubstituted hydroxyapatite. In: Handbook of Ionic Substituted Hydroxyapatites. Elsevier: London, UK;2020:237-257

10 Wang L, Xie X, Li C, et al. Novel bioactive root canal sealer to inhibit endodontic multispecies biofilms with remineralizing calcium phosphate ions. J Dent 2017;60:25-35

11 Kececi AD, Ureyen Kaya B, Adanir N. Micro push-out bond strengths of four fiber-reinforced composite post systems and 2 luting materials. Oral Surg Oral Med Oral Pathol Oral Radiol Endod 2008;105(01):121-128

12 Rahoma A, AlShwaimi E, Majeed A. Push-out bond strength of different types of mineral trioxide aggregate in root dentin. Int J Health Sci (Qassim) 2018;12(05):66-69
13 Al-Haddad AY, Kutty MG, Che Ab Aziz ZA. Push-out bond strength of experimental apatite calcium phosphate based coated gutta-percha. Int J Biomater 2018;2018:1731857

14 Lee JK, Kwak SW, Ha J-H, Lee W, Kim H-C. Physicochemical properties of epoxy resin-based and bioceramic-based root canal sealers. Bioinorg Chem Appl 2017;2017:2582849. Doi: 10.1155/2017/ 2582849

15 Collares FM, Leitune VC, Rostirolla FV, Trommer RM, Bergmann CP, Samuel SM. Nanostructured hydroxyapatite as filler for methacrylate-based root canal sealers. Int Endod J 2012;45(01):63-67

16 Bae WJ, Chang SW, Lee SI, Kum KY, Bae KS, Kim EC. Human periodontal ligament cell response to a newly developed calcium phosphate-based root canal sealer.J Endod 2010;36(10):1658-1663

17 Baras BH, Sun J, Melo MAS, et al. Novel root canal sealer with dimethylaminohexadecyl methacrylate, nano-silver and nanocalcium phosphate to kill bacteria inside root dentin and increase dentin hardness. Dent Mater 2019;35(10):1479-1489

18 Zafar MS, Alnazzawi AA, Alrahabi M, Fareed MA, Najeeb S, Khurshid Z. Nanotechnology and nanomaterials in dentistry. In: Advanced Dental Biomaterials. Elsevier London, UK2019:477-505

19 Witjaksono W, Naing L, Mulyawati E, Samsudin A, Oo MMT. Sealing ability of hydroxyapatite as a root canal sealer: in vitro study. Dent J (Maj Ked Gigi) 2007;40(03):101-105

20 Toledano M, Muñoz-Soto E, Aguilera FS, et al. The mineralizing effect of zinc oxide-modified hydroxyapatite-based sealer on radicular dentin. Clin Oral Investig 2020;24(01):285-299

21 Akman M, Akman S, Derinbay O, Belli S. Evaluation of gaps or voids occurring in roots filled with three different sealers. Eur J Dent 2010;4(02):101-109

22 Toledano M, Muñoz-Soto E, Aguilera FS, et al. A zinc oxidemodified hydroxyapatite-based cement favored sealing ability in endodontically treated teeth. J Dent 2019;88:103162

23 Ali S, Sangi L, Kumar N, Kumar B, Khurshid Z, Zafar MS. Evaluating antibacterial and surface mechanical properties of chitosan modified dental resin composites. Technol Health Care 2020;28(02): 165-173

24 Mohammadian F, Farahanimastary F, Dibaji F, Kharazifard MJ. Scanning electron microscopic evaluation of the sealer-dentine interface of three sealers. Iran Endod J 2017;12(01):38-42

25 Ungor M, Onay EO, Orucoglu H. Push-out bond strengths: the Epiphany-Resilon endodontic obturation system compared with different pairings of Epiphany, Resilon, AH Plus and gutta-percha. Int Endod J 2006;39(08):643-647 